\title{
A Review on Cable-driven Parallel Robots
}

CrossMark

\author{
Sen Qian ${ }^{1}$, Bin Zi ${ }^{1 *}$, Wei-Wei Shang ${ }^{2}$ and Qing-Song $X u^{1,3}$
}

\begin{abstract}
Cable-driven parallel robots (CDPRs) are categorized as a type of parallel manipulators. In CDPRs, flexible cables are used to take the place of rigid links. The particular property of cables provides CDPRs several advantages, including larger workspaces, higher payload-to-weight ratio and lower manufacturing costs rather than rigid-link robots. In this paper, the history of the development of CDPRs is introduced and several successful latest application cases of CDPRs are presented. The theory development of CDPRs is introduced focusing on design, performance analysis and control theory. Research on CDPRs gains wide attention and is highly motivated by the modern engineering demand for large load capacity and workspace. A number of exciting advances in CDPRs are summarized in this paper since it is proposed in the 1980s, which points to a fruitful future both in theory and application. In order to meet the increasing requirements of robot in different areas, future steps foresee more in-depth research and extension applications of CDPRs including intelligent control, composite materials, integrated and reconfigurable design.
\end{abstract}

Keywords: Cable-driven parallel robots, Design and modelling, Control and planning, Performance and optimization

\section{Introduction}

Cable-driven parallel robots (CDPRs) are known as a type of parallel robots. In CDPRs the end-effector (EE) is suspended by several flexible cables, taking the place of rigid links in traditional rigid-link parallel robots. Compared with traditional rigid-link parallel robots, CDPRs have much smaller inertia and higher payload to weight ratio, which provides high speed and acceleration of the EE [1-4]. In addition, due to the extension range and flexibility of cables, CDPRs can be applied in challenging tasks that require motivation with large reachable workspace and better flexibility as well [5-8].

Research on CDPRs originates from America in 1984. A cable-controlled parallel manipulator is designed for underwater operation. In 1989, the RoboCrane project is started in America by the National Institute of Standards and Technology (NIST), which stand out for simplicity and extensive use especially in processing machinery, port cargo handling, bridge construction, welding and other areas, as shown in Figure $1[9,10]$.

In the late 1980s, August Design Company developed a video tape recorder system named SkyCam with 4 cables

\footnotetext{
*Correspondence: binzi.cumt@163.com

${ }^{1}$ School of Mechanical Engineering, Hefei University of Technology, Hefei 230009, China

Full list of author information is available at the end of the article
}

and up to $44.8 \mathrm{~km} / \mathrm{h}$ maximum speed, which is widely used for live broadcast in large scale, especial for highspeed tracking photography, as shown in Figure 2 [11]. Duan et al. [12] from Xidian University proposed a novel feed cable-driven structure for feed support system in 500-m aperture spherical radio telescope (FAST) in 1999 in China, in order to move the feed cabin of large spherical radio telescope, as shown in Figure 3. Integrated mechanical and electronic designing, as well as cooperative control technology make FAST one of the most successful applications of CDPRs, which is located in the southwest of China [13].

In the last decades, research on CDPRs gains wide attention and is highly motivated by the modern engineering demand for large load capacity and workspace. CDPRs have been increasingly and widely applied in relevant tasks, such as construction, rescue systems, rehabilitation, and even three-dimensional print.

For instance, a cooperative CDPR consists of multiple mobile cranes is designed, as shown in Figure 4. The cooperation problems is considered and analyzed, including the localization of multiple mobile cranes, obstacle avoidance and adaptive orientation control of the payload [14]. Seriani et al. [15] proposed a modular CDPR deployed by a rover shown in Figure 5. Due to the large work scale of CDPRs, the mentioned modular 

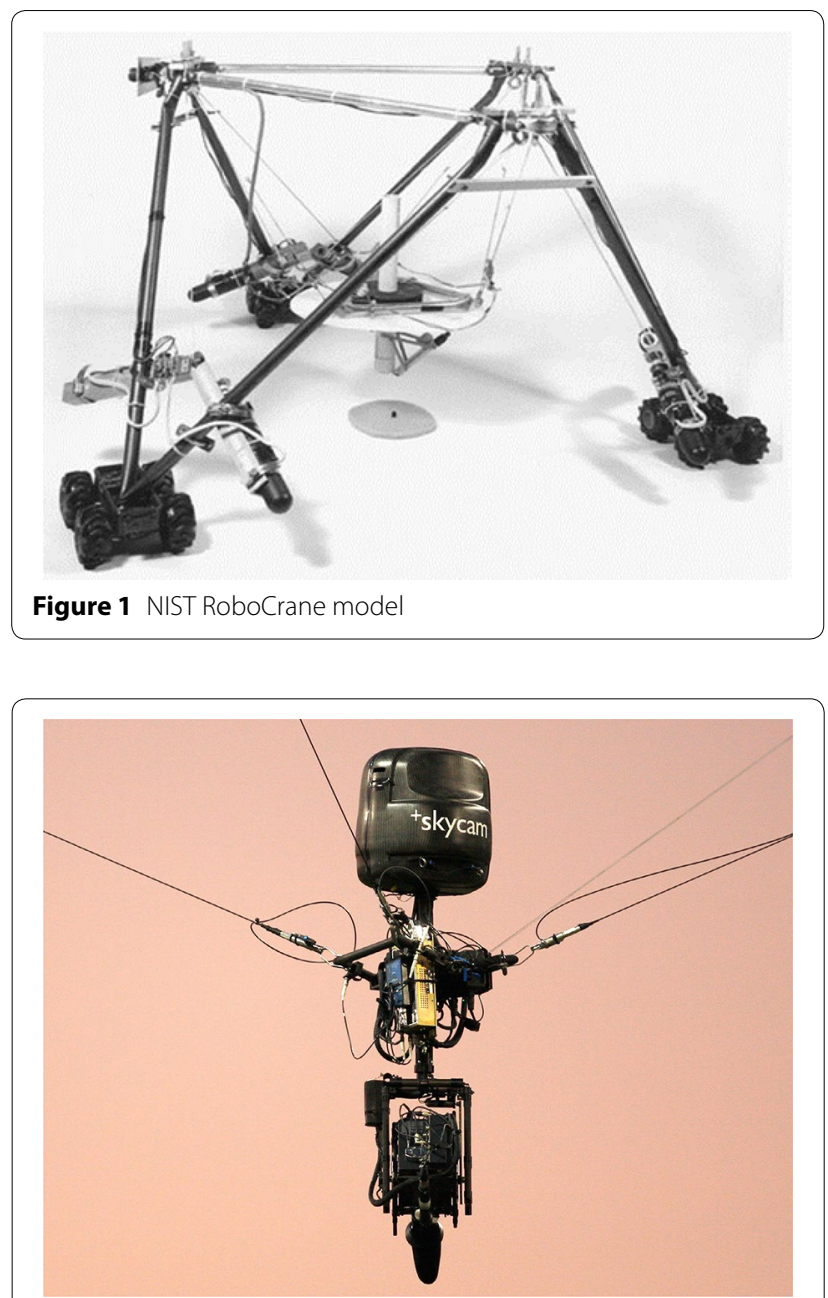

Figure 2 Cable-driven SkyCam video tape recorder system

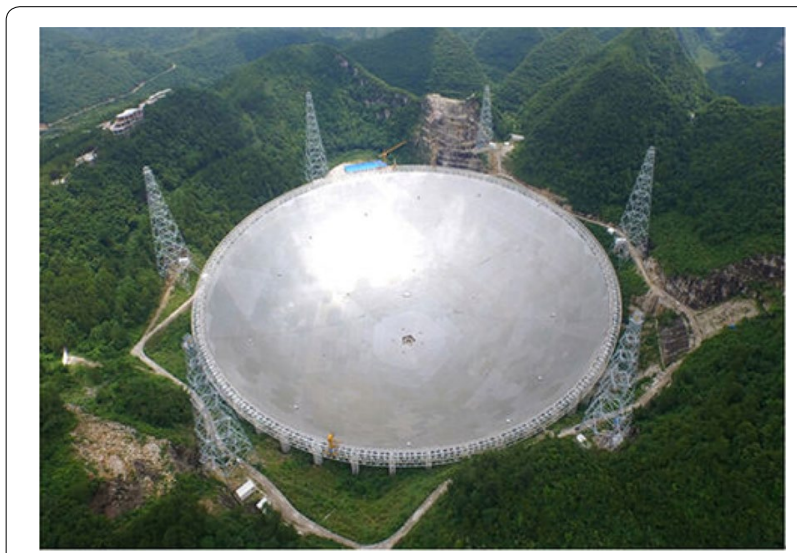

Figure 3 Large spherical radio telescope

CDPR can be applied in inspection tasks in field and rugged environment.

Varela et al. [16] presented an experimental characterization of the biomechanics of human gait by means

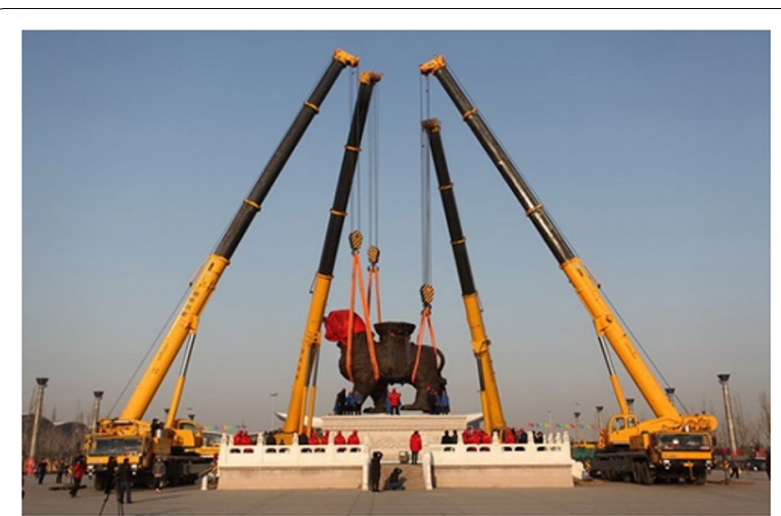

Figure 4 CDPR of cooperative multiple cranes

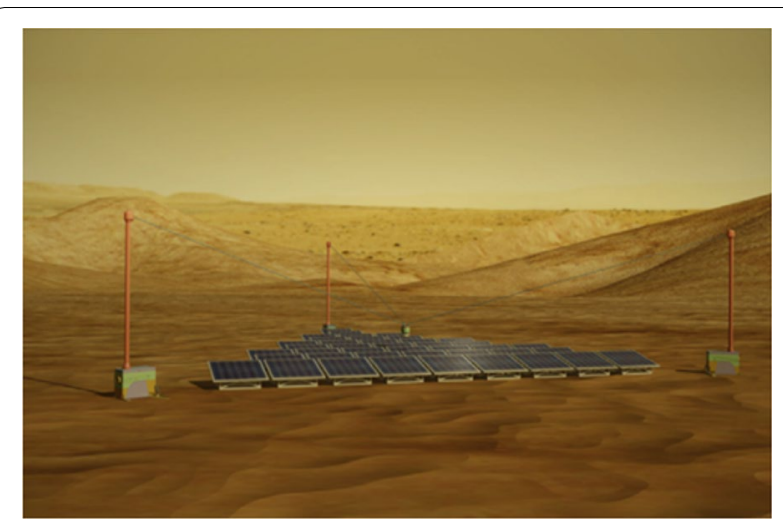

Figure 5 Modular CDPR for solar collection in field and rugged environment

of a CDPR named Cassino tracking system, which is a low-cost operable system used as an assessing device for diagnosis and rehabilitation procedures in certain clinical application, as shown in Figure 6.

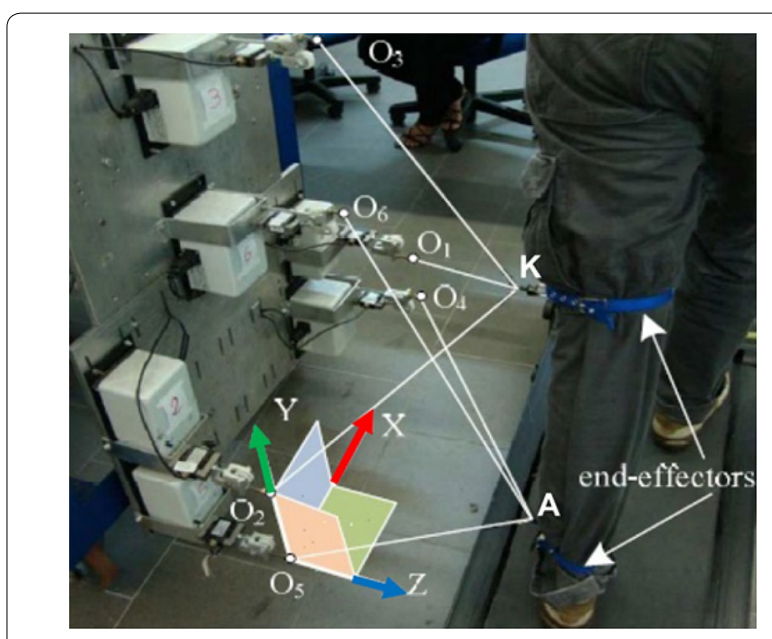

Figure 6 A treadmill gait assessment of Cassino tracking system 
Mao et al. [17] proposed a cable-driven exoskeleton for upper arm neural rehabilitation shown in Figure 7. The arm neural rehabilitation exoskeleton can provide more natural training experience driven by multi-stage CDPRs rather than rigid links. Pinto et al. [18] presented a CDPR called SPIDERobot with four degrees of freedom (DOFs) for automated architectural construction, as shown in Figure 8 . The rotating claw can pick construction material with desired orientations, which is driven by four cables. Barnett and Gosselin [19] introduced a cable-driven 3D printer with large workspace rather than traditional 3D printers driven by rigid links, shown in Figure 9, which utilizes a 6-DOF CDPR for positioning and provides large workspace of motion. Due to the geometric feedback
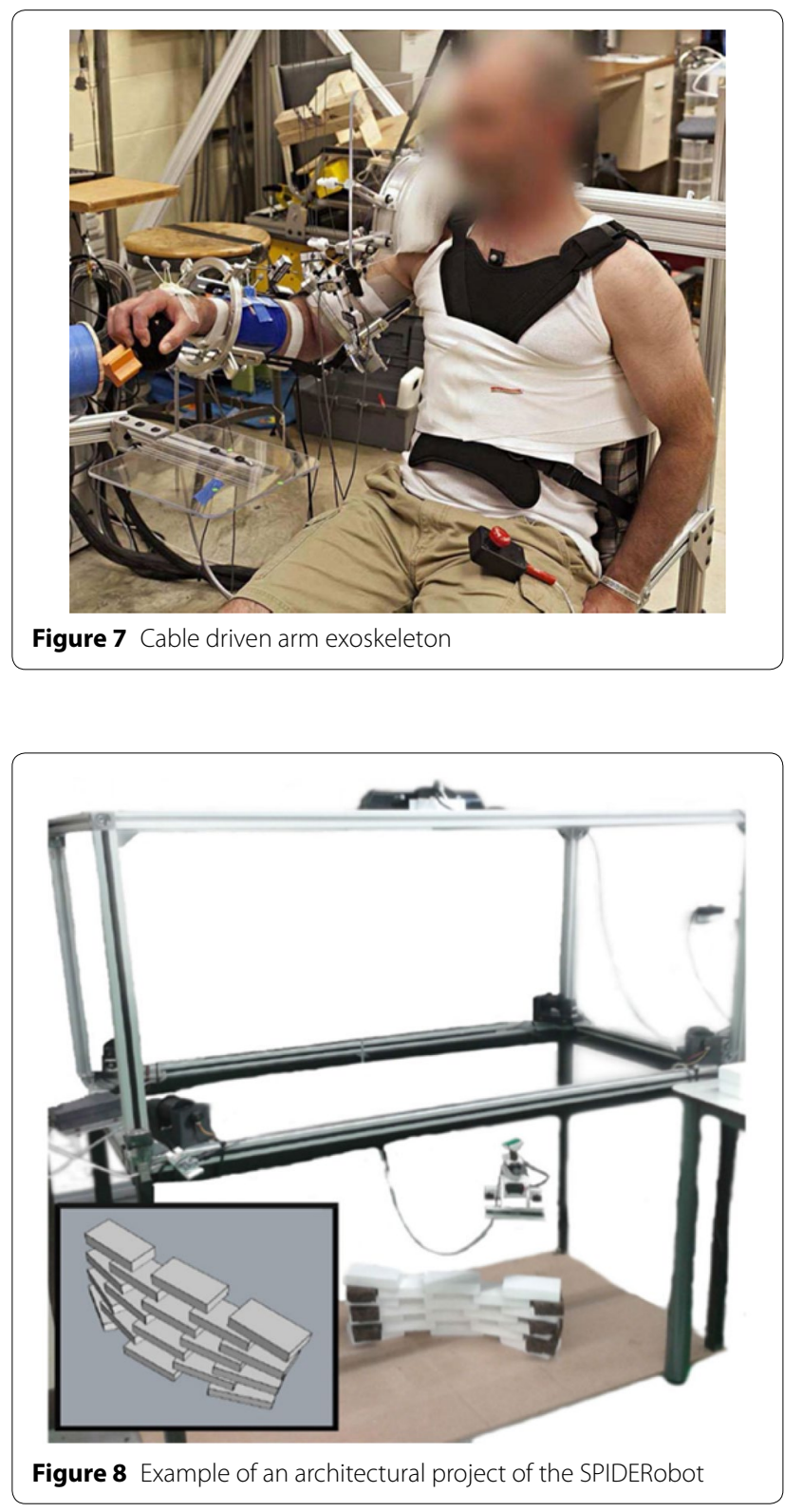

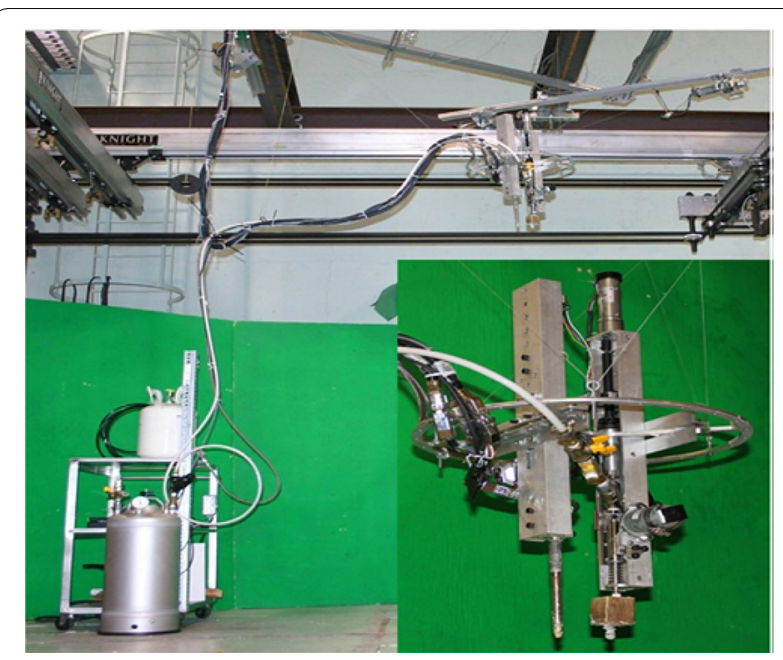

Figure 9 Hybrid-driven-based cable parallel mechanism

control system, the stability and accuracy of the CDPR 3D printer are enhanced.

There is much prior work in analysis and application of CDPRs. In the last decades, research on CDPRs focus on the following aspects, including design and modelling, performance and optimization, control and planning.

\section{Design and Modelling \\ 2.1 Design}

A typical CDPR system is made up of three parts, including a fixed platform, a mobile platform and several cables, which are used to connect the fixed platform and mobile platform. The cable length can be changed through winches actuated by motors installed in the fixed platform, as shown in Figure 10 [20, 21].

Due to the advantages of CDPRs, including small moving inertia and large workspace of motion, more and more CDPRs with novel structures and functions have

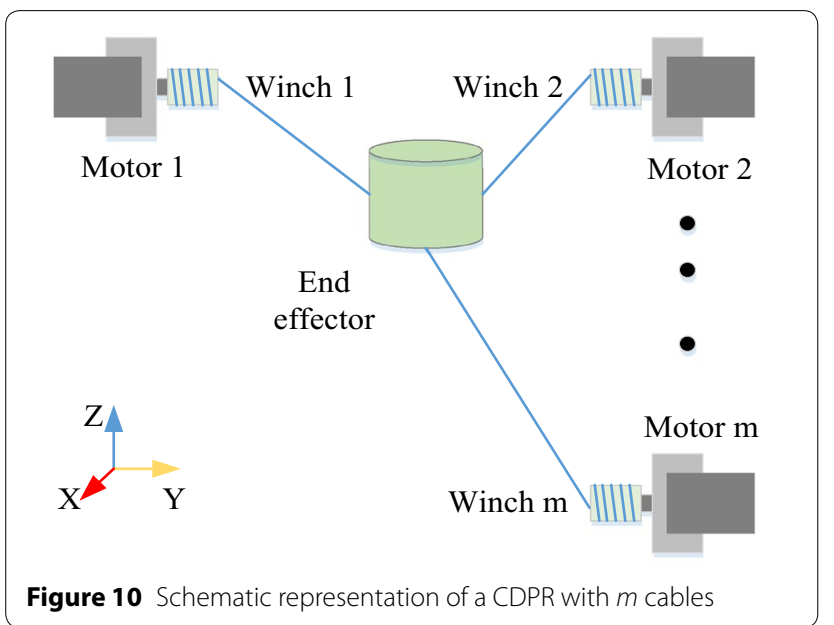


been developed more recently. An important characteristic of CDPRs is well known as cables can be only driven by positive tension in order to keep the straight line shape rather than negative compression. A CDPR is under-constrained if the position and orientation of the $\mathrm{EE}$ in the robot is determined only by its gravity. While if the position and orientation of the $\mathrm{EE}$ is completely determined by the lengths of the cables, the CDPR is fully or redundantly constrained. Generally speaking, CDPRs with $n$ DOFs driven by $\mathrm{m}$ cables can be classified into three types according to the mobility and statics: underconstrained CDPRs when $n+1>m$, fully constrained CDPRs when $n+1=m$, and redundantly constrained CDPRs when $n+1<m$, respectively [22].

For fully and redundantly constrained CDPRs, the position and orientation of the EE only depends on kinematic and static. For instance, Liu et al. [23] introduced two novel architectures of planar CDPRs with spring. Actuation redundancy is not required with spring-loaded mechanisms. Azizian and Cardou [24, 25], solved the dimensional synthesis problem in order to find two fully constrained planar and spatial CDPRs with a prescribed workspace contained in wrench-closure workspace. Gagliardini et al. [26] dealt with a reconfigurable CDPR with movable cable connection points. The mentioned CDPR is more suitable and flexible in complex environment where cable collisions with obstacles cannot be avoided within the workspace of a CDPR with fixed cable connection points. $\mathrm{Zi}$ et al. [27] proposed and analyzed a winding hybrid-driven CDPR, combining the advantages of both planar five-bar hybrid-driven mechanism and CDPR.

While for under-constrained CDPRs, the EE tends to approach the position and orientation of minimum gravitational potential energy. Under-constrained CDPRs rely on gravity to determine the position and orientation of the EE, whose state can be easily changed by any external disturbances. For instance, Kumar [28] analyzed the kinematics problem of under-constrained CDPR consists of multiple aerial robots, as shown in Figure 11. The payload is suspended by three robots with three cables.

Donohoe et al. [29] presented a planar under-constrained CDPR as a force optimal mechatronic device with the purpose of operating on the large scale vertical plane. The pulley arms provide redundant DOFs, which improve the ability of CDPR in generating force. However, in spite of the coupling between the kinematics and statics of the robot, under-constrained CDPRs are much simpler in structure rather than fully and redundantly constrained CDPRs driven by at least six or seven cables. Capua et al. [30] presented a mobile CDPR with novel structure named SpiderBot, which consists of four cable mechanisms, a mobile platform, dispensing

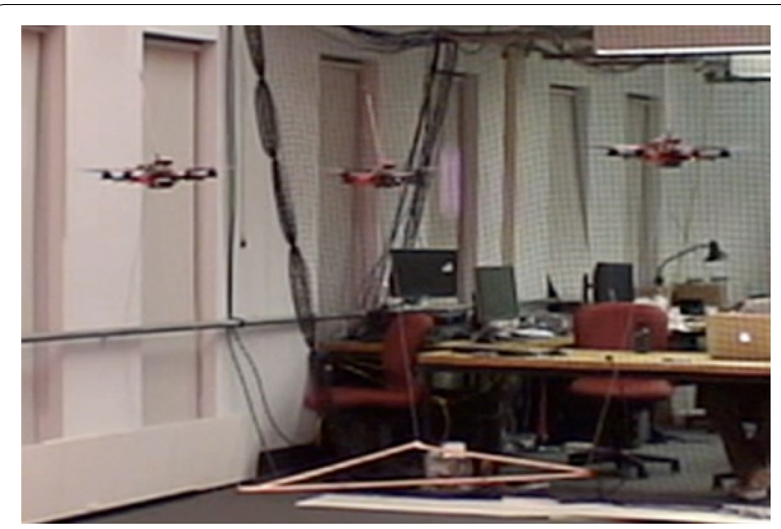

Figure 11 Under-constrained CDPR of multiple aerial robots

mechanisms, rolling mechanisms and grippers at the end of the cable. Qian et al. [31] proposed a CDPRs for multiple mobile cranes. Three cables are used to move the payload together, with better stability and safety rather than single crane operation. In order to enlarge the operation scale of motion and overcome the drawback of CDPRs in terms of the potential collisions between the robot and obstacles in the workspace, $\mathrm{Zi}$ et al. [32] presents a reconfigurable CDPR with a circular orbit. The spatial topology of the reconfigurable CDPR can be reconfigured through managing the branch numbers and the cable drawing points of the EE, as shown in Figure 12.

The key characteristic of CDPRs is that cables can be only driven by positive tension in order to keep the straight line shape rather than negative compression, which limits the development and application of CDPRs. In order to overcome the shortcoming, more and more novel design are proposed though structure synthesis in the last decades combining CDPRs with other mechanisms, including grid links, springs, flexure hinge and other mechanisms or actuators, such as shape memory alloy and pneumatic artificial muscles.

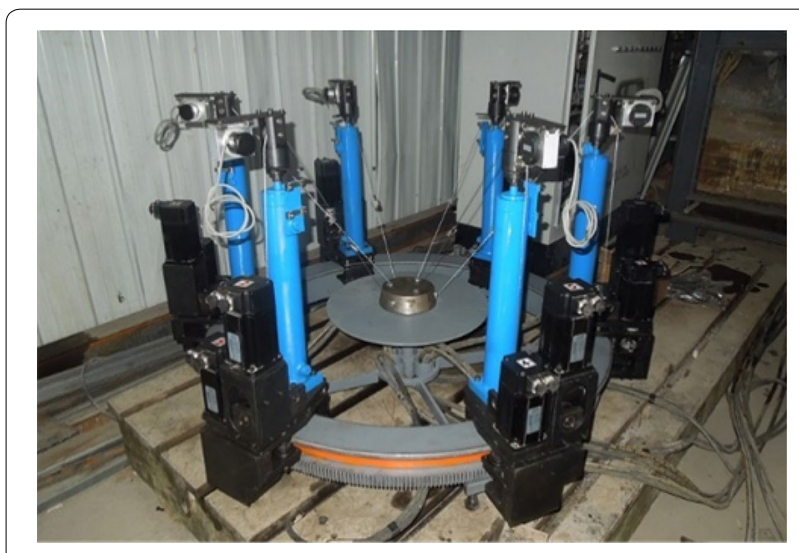

Figure 12 Reconfigurable CDPRs with a circular orbit 
Structure synthesis is a systematic and efficient way for mechanism design. Though structure synthesis, one can obtain different types of mechanisms according to desired number of links and DOFs, and it. For instance, Dong et al. [33] proposed a novel design of a snake arm robot with twin actuation construction. Due to the compliant joint construction, the robot has a great flexibility and an appropriate stiffness. Suh et al. [34] proposed the innovative pulleyless rolling joint, which can avoid slack and unwanted bending of cables with elastic fixtures. Cui et al. [35] designed a CDPR exoskeleton with 7 DOFs for dexterous motion training of the whole arm, including an additional wrist module and eight cables routed through the exoskeleton cuffs to realize the motion of the whole arm. Mao and Agrawal [36] presented a CDPR upper arm exoskeleton with 5 DOFs. The workspace of the CDPR exoskeleton can be optimized through the adjustable cable routing points. Zhao et al. [37] designed a CDPR in order to imitate the motion of human necks. In the mentioned CDPR, a pneumatic muscle active support is applied to imitate the spinal muscles, and cable actuators imitate neck muscles, respectively. Gao et al. [38] presented a low motion-noise humanoid head/neck simulator driven by seven cables, which can generate 1-DOF jaw movement and 3-DOF neck movement. A generalized model of multi-link CDPRs is presented by Lau et al. [39]. The kinematics and dynamics model of the multilink CDPR are established allowing for arbitrary cable routing. The inverse dynamics analysis is carried out for an 8-link and 24-DOF neck model actuated by 76 cables, as shown in Figure 13.

\subsection{Kinematics and Dynamics}

Compared with inverse kinematic problems of series robots, the inverse kinematic problems of parallel robots is easier. Inversely, the forward kinematic problems of parallel robots become more difficult. Gao et al. [40] presented a novel bio-inspired CDPR with a flexible spine.
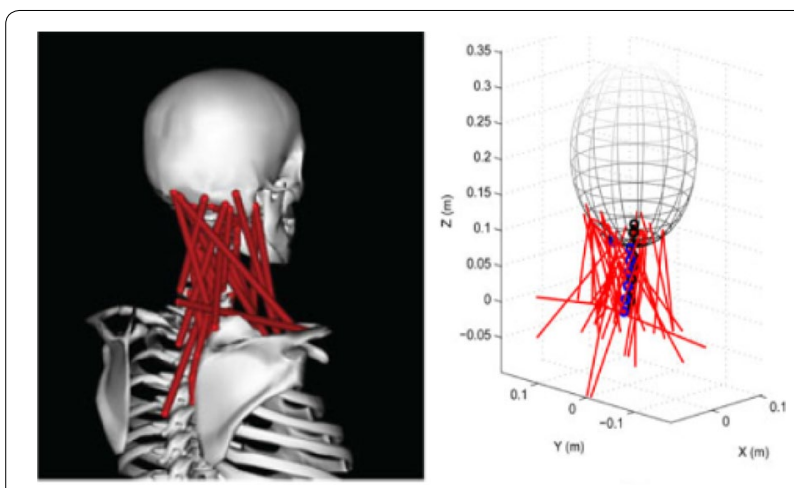

Figure 13 8-link 24-DOF neck model actuated by 76 cables
In order to minimize the tension actuating on the cables, optimization of the cable placements are carried out by combing the bending statics of spring and torque balance equations. Based on interval analysis, Berti et al. [41] presented an efficient algorithm for solving the direct geometrico-static problem of under-constrained CDPRs. The tests conducted have indicated that accurate results can be obtained with the mentioned algorithm, regardless of the accuracy of cable model.

For under-constrained CDPRs, kinematics and statics must be analyzed simultaneously because they are coupled. Carricato and Merlet [42, 43], established the direct geometrico-static modeling of a 3-DOF underconstrained CDPRs and present an effective procedure for elimination. With this method, one can obtain the least degree univariate polynomial free of spurious factors when dealing with the coupled kinematics and statics problem of under-constrained CDPRs. Jiang and Kumar [44] presented a CDPR consisting of multiple aerial robots, which can be used for cooperative transport of payloads. The kinematic model of the CDPR is established on the basis of dialytic elimination, which is used to determine the position and orientation of each aerial robot as well as the payload.

CDPR is a feasible way to achieve motion in large workspace. However, inevitable vibrations and sagging of long cables dramatically reduce the positioning accuracy in large workspace applications, which cannot be neglected during the dynamic modelling of CDPRs. The dynamic model can be established with different approaches, such as Lagrange equation, Newton-Euler equations, Kane equation, Udwadia-Kalaba equation, principle of virtual work, etc. [45-48]. For instance, Du et al. [49] addressed dynamic modeling of large CDPRs on basis of a variable domain finite element method. The influences of cable length and mass variation are both taken into account. In conventional researches, cables in CDPRs are usually treated as simple linear elements for simplicity, which cause the inaccuracy of cable modelling. To overcome the shortcoming, a dynamic model for CDPRs is presented considering the slowly time-varying length of cables in Ref. [50]. Khosravi and Taghirad [51] discussed the dynamics of a fully-constrained CDPRs with elastic cables, considering longitudinal vibration of cables when establishing the dynamic model. For multilink CDPRs, Joint interaction forces and moments cannot be ignored, which is considered for the first time according to the objective function and constrains in inverse dynamics of multi-link CDPRs [52]. Wang et al. [53] proposed a new three dimensional dynamics of cable-driven soft robot by combining the geometrically exact Cosserat rod theory and Kelvin model, which is validated by comparison 
between the numerical results in both two and three dimensional cases.

\section{Performance and Optimization}

Performance analysis plays an important role as the fundamental tools in optimal design of CDPRs, including workspace, stiffness, sensitivity, etc. [54-56]. Cables can only exert tension, namely unilateral actuating property, traditional performance analysis methods for research on rigid-link robots can hardly applied directly in CDPRs. Thus, various analysis methods on performance of CDPRs were proposed in the last decades.

Due to the larger extension range of cables rather than rigid links, the workspace of CDPRs becomes larger than that of rigid-link parallel robots. The force-closure workspace of CDPRs is defined as a set of positions where the cable tensions can balance arbitrary external forces exerted on the EE. Since cables can only pull rather than push the EE, it is usually hard to meet the desire requirements for the wrench-feasible workspace of CDPRs. The workspace of CDPRs can be obtained with different numerical generation methods presented in many literatures [57-60]. Taking a planar CDPR as the object, Azizian et al. [61] proposed a graphical method in order to generate the constant-orientation wrench-closure workspace. Via installing springs connecting the fixed platform and the $\mathrm{EE}$, the constant-orientation wrenchclosure workspace can be adjusted [62]. The influences of spring parameters on CDPR workspace are analyzed. On the basis, the optimization is carried out to obtain the feasible parameters of spring. Ouyang and Shang [63] developed a new computation method to generate the force-closure workspace of CDPR. The linear matrix inequalities are solved and the null space of the matrix of the robot is derived.

Positional accuracy and load capacity of the EE rely on high stiffness of the robot in practice engineering. For CDPRs, the stiffness is depended on the stiffness of cables as well as the internal tension exerted on the cables. Although the flexible property of cables can increase the flexibility of CDPRs, the low stiffness of cables should be considered which limits the accuracy of the whole robot system. Yeo et al. [64] introduced a CDPR with tension resolution equipment, which is able to effectively regulate stiffness of the whole CDPR, as shown in Figure 14. There is a novel variable stiffness device installed along each cable. Yuan et al. [65] analyzed the dynamic stiffness of CDPR through identifying the natural frequencies of the system. The static stiffness of CDPRs was evaluated according to the variation of the EE. Arsenault [66] evaluated the stiffness throughout the workspace of a spatial 3-DOF CDPR. In addition, the intuitive stiffness indices

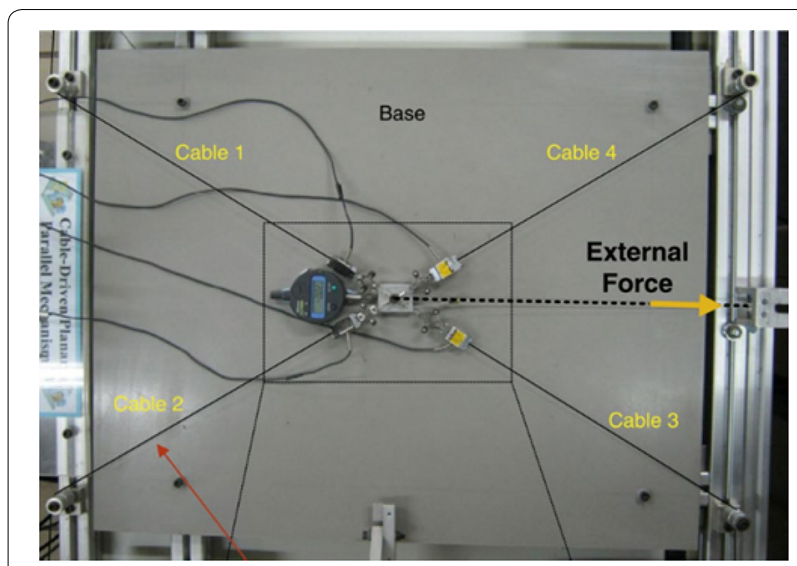

Figure 14 Two-DOF planar CDPRs prototype with variable stiffness device

are mapped and extracted on the basis of the stiffness matrix.

The accuracy is one of the most important indicators that should be considered for design and control of a CDPR. There are many factors that would reduce the accuracy of CDPRs, such as design tolerance, assembly error, thermodynamic error, control response, etc. The influence of these error sources can be investigated via error modelling and sensitivity analysis, which can assist the designer to establish the mapping relation graph between the pose error of the EE and these error sources. On the basis of sensitivity analysis, the pose error of the EE can be minimized through precision machining and assembly. The error model of CDPRs can be established with different algorithms, such as the matrix differentiation method, perturbation method, vector analyzing method, etc. In Ref. [67], large-scale 3D printing with cable-driven parallel robots are presented with acceptable accuracy amplitude $(<4 \mathrm{~mm})$ in horizontal plane in a maximum workspace of $13.6 \mathrm{~m} \times 9.4 \mathrm{~m} \times 3.3 \mathrm{~m}$. In order to simplify the formulation of the calibration algorithms, Chen et al. [68] derived the error model of a humanoidarm CDPR with passive spherical joint based on inverse kinematics. The accuracy of the humanoid-arm CDPR is obviously improved and the pose errors of the $\mathrm{EE}$ are reduced to less than $2 \mathrm{~mm}$ after the self-calibration algorithm based on error analysis, as shown in Figure 15.

As mentioned, the performance analysis is conducted aiming at optimization of CDPRs design. The best performance in terms of workspace, stiffness, sensitivity, etc., should be considered to obtain the feasible parameters that meet the requirement of different tasks. For instance, Jamshidifar et al. [69] developed a general model for redundant-constrained CDPRs, as shown in Figure 16. External disturbances are exerted in the desired 


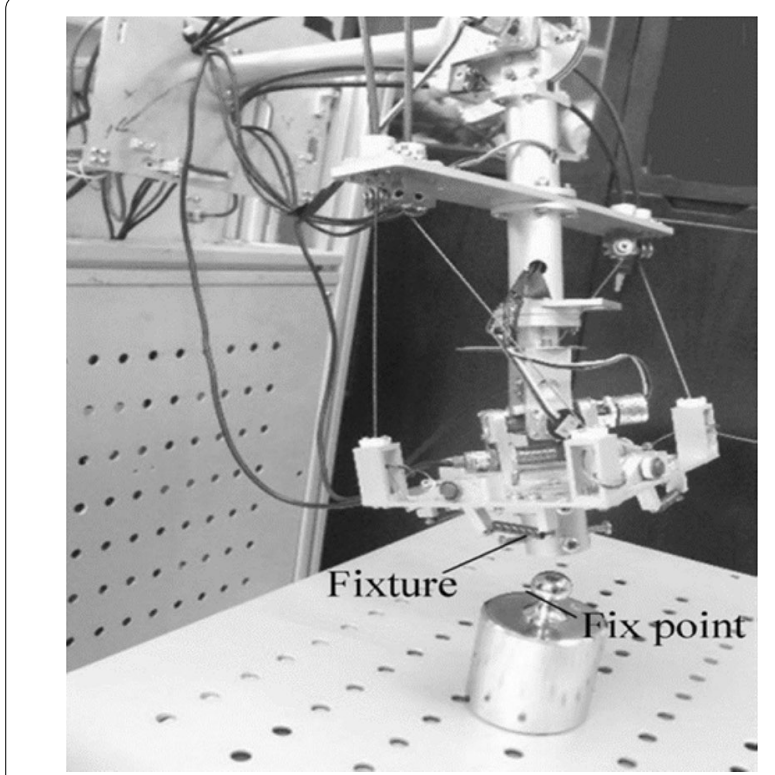

Figure 15 Prototype of CDPR with passive spherical joint

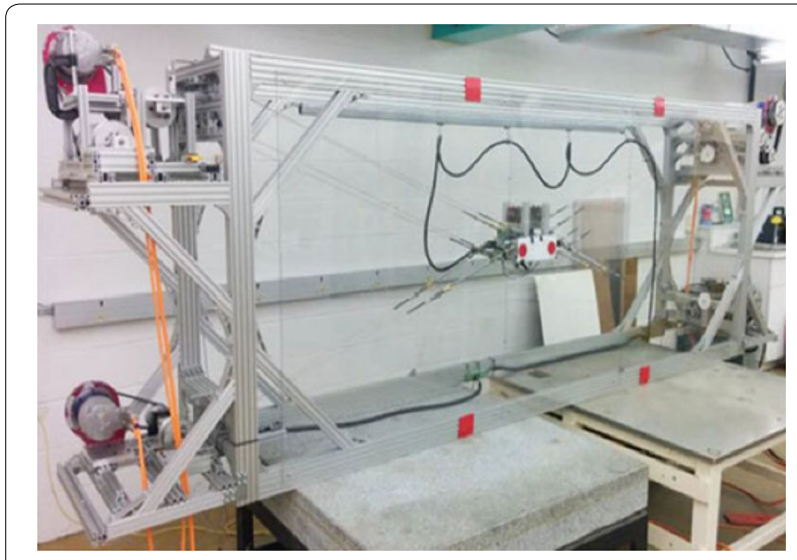

Figure 16 Prototype of planar warehousing CDPR

direction, stiffness optimization is conducted in order to minimize the unwanted perturbations. The stiffness and dexterity of CDPRs can be presented based on stiffness and Jacobian matrices. By adjusting structural parameters and the sectional area of cable, Du et al. [70] presented an optimization model in order to improve the stiffness and dexterity. Bryson et al. [71] proposed a generalized method for optimal design of the workspace of a CDPR leg with stochastic method, as shown in Figure 17. With the purpose of improving the mechanical performance of CDPRs and maximizing the orientation wrench-feasible workspace, Ouyang and Shang [72] optimized the distribution of the winches on the fixed platform as well as the hinges on the mobile platform, respectively.

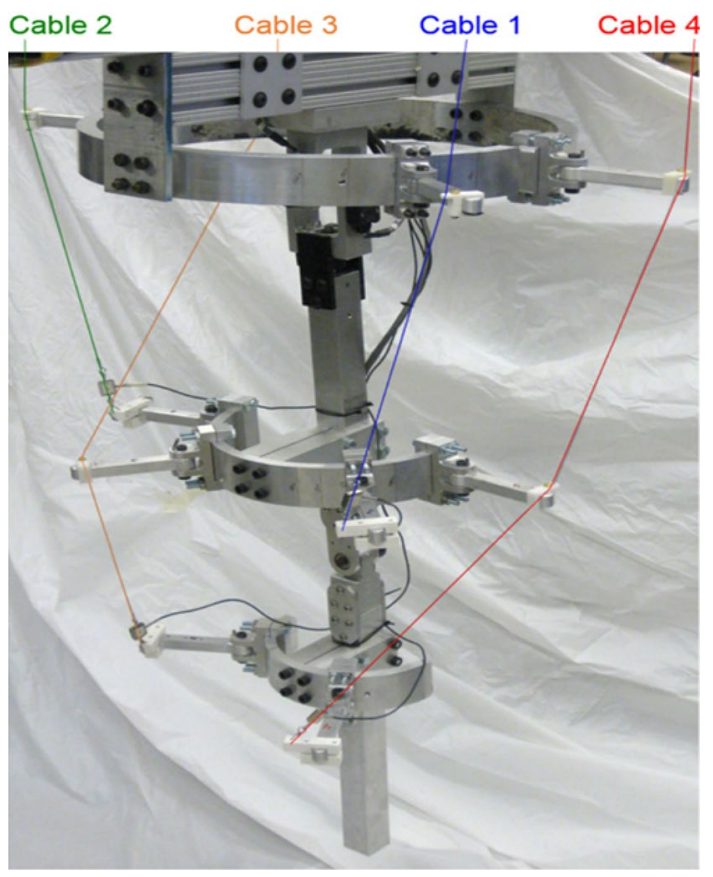

Figure 17 Prototype of cable-driven robot leg

\section{Control and Planning}

\subsection{Control Theory}

Substituting cables for rigid links introduces inevitable challenges for the control of CDPRs, compared with that of traditional rigid-link parallel robots. In addition, it is difficult to control the position and orientation of the EE precisely for its low stiffness. Due to the mentioned physical limitation that endure tension but not compression, some widely used control methods cannot applied in CDPRs directly, which must be modified to meet the special property of cables. In comparison with the large number of studies about rigid-link parallel robots, few has been published on the control of CDPRs. Researches on CDPRs all over the world have applied some control algorithms in CDPRs, including sliding mode control, hybrid position/force control, adaptive control, etc. Several efforts had been exerted on control of CDPRs for real-time and accuracy purposes [73-76].

For instance, Yang et al. [77] developed an adaptive controller for a rehabilitation CDPR. The mentioned controller with a fuzzy tuner can adjust and optimize the time-varying control parameters on the basis of position error. The control objectives of the CDPR have been realized based on heuristics and prior knowledge, including monotonicity and symmetry [78], as shown in Figure 18.

Babaghasabha et al. [79] presented an adaptive robust controller for a fully-constraint CDPR according to the upper bound of the uncertainties. This sliding mode 


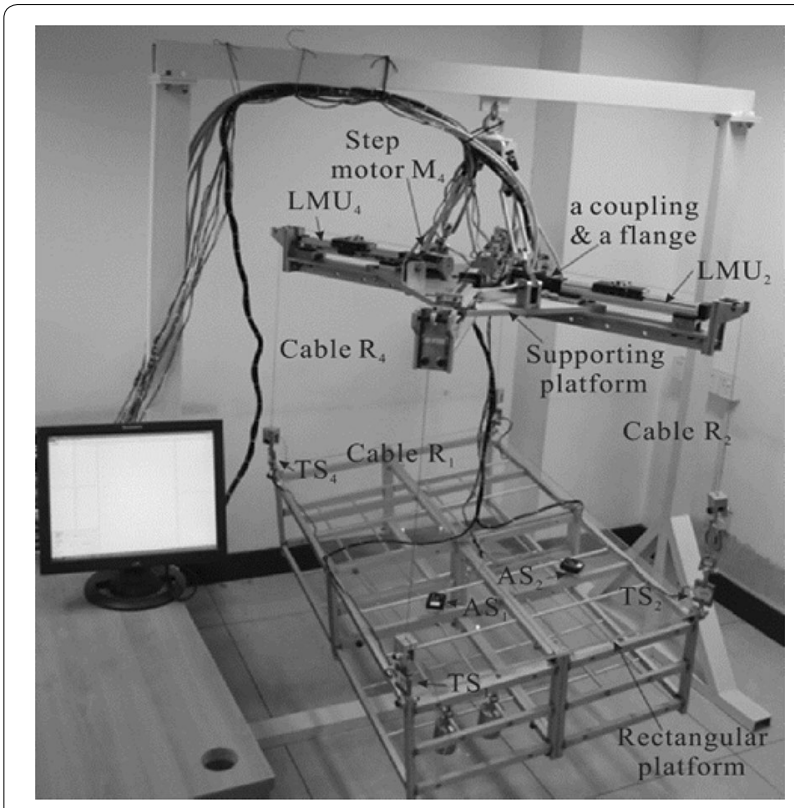

Figure 18 CDPR with adaptive fuzzy control scheme

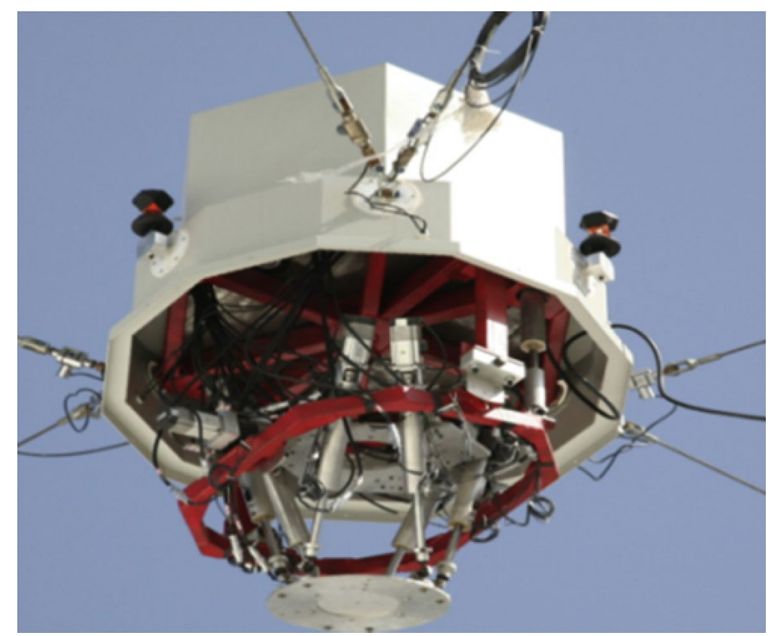

Figure 19 1:15 similarity prototype of feed support system in FAST

controller can work without prior knowledge as well as the linearization of dynamic models. Abdelaziz et al. [80] presented a position control method for CDPR. An internal cable tension control loop is introduced into the controller for compensating friction. Particular design constraints were considered including the length, size and materials of transmissions. Tang et al. [81] presented a hybrid position/force controller for CDPRs. The pseudo-drag problem of flexible cable is taken into account and prevented. The approach is validated in 1:15 similarity prototype of feed support system in FAST, as shown in Figure 19.

\subsection{Trajectory Planning}

One of the major drawback of CDPRs is the cable sagging during the moving of EE. It is a challenging problem to solve the trajectory planning of CDPRs, due to the pseudo-drag problem of cables [82-84]. Thus, compared with that of traditional robots, the analyses of the trajectory generation for CDPRs are completely different. For fully constrained CDPRs, the fact that all the DOFs of the EE can be controlled makes the trajectory planning problem easier. The force-closure workspace can be applied to avoid pseudo-drag of cables during operation. However, for under-constrained CDPRs, the controllable workspace does not exist, increase the difficulty during the trajectory planning of under-constrained CDPRs.

Several contributions presented in literature have dealt with the trajectory planning of CDPRs. For instance, in order to solve the point-to-point motion of a 3-DOF CDPR, Jiang and Gosselin $[85,86]$ proposed a dynamic trajectory planning method. Consecutive points can be connected with the calculated trajectories in sequence which are located outside of the static workspace of the CDPR. Zhang and Shang $[87,88]$ proposed a geometrical approach for trajectory planning of a spatial under-constrained CDPR with 3 DOFs. According to the geometric properties of the cable tension constraints, the periodic trajectory parameters can be calculated. Taking a planar 2-DOF redundantly actuated CDPR as the object, Tang et al. [89] analyzed the dynamic trajectory planning on the basis on periodic trajectory and antipodal theory. In order to obtain maximum dynamic load capacity of a spatial under constrained CDPR, a geometrical based variational optimization method was proposed in Ref. [90].

\section{Conclusions}

In this paper, the history of the development of CDPRs is introduced and several successful latest application cases of CDPRs are presented. The development of CDPRs is presented focusing on design, performance analysis and control theory with the purpose of assisting readers to obtain a detail and quick overview on the design and analysis of CDPRs.

In contrast with classical rigid-link parallel robots, CDPRs are driven by flexible cables rather than rigid links to control the position and orientation of the EE. CDPRs exhibit advantages of parallel robots compared with serial robots including higher load-weight ratio. Moreover, CDPRs can provide many other new desirable characteristics, including high speed and acceleration, high payload-to-weight ratios, and potentially large workspace. However, different from rigid links, cables can only exert tension, namely unilateral actuating property, which limits the development and application of 
CDPRs. Thus, traditional methods for research on rigidlink robots can hardly applied directly in CDPRs. In order to overcome the shortcoming, research on CDPRs focus on the following aspects, including design and modelling, performance and optimization, control and planning. Owing to the development in optimal design and control theory in last decade, CDPRs have been significantly improved in terms of kinematic and dynamic performance, and increasingly applied to more and more relevant tasks, including engineer, astronomy, bionics, etc. However, CDPRs are still rarely applied industrial manufacturing in contrast with serial robots and rigid link parallel robots.

There are a number of exciting advances in CDPRs in recent years, which points to a fruitful future. In order to meet the increasing requirements of robot in different areas, future steps foresee more in-depth research and extension applications of CDPRs. First, the integrated design of CDPRs of different configurations with better performances should be carried out with the type synthesis theory. Second, more advanced controller and actuator can be applied in CDPRs for higher trajectory tracking performance. Third, stiffness and load-capacity of CDPRs can be improved though the combination with new composite materials. In addition, the concept of reconfigurable and modular design has been widely applied in series robots and rigid-link parallel robots successfully, which can be generalized to the design of CDPRs for better environmental suitability, flexibility and cost performance.

\section{Authors' Contributions}

BZ was in charge of the whole trial; SQ wrote the manuscript; WWS and QSX assisted with structure and language of the manuscript. All authors read and approved the final manuscript.

\section{Author details \\ ${ }^{1}$ School of Mechanical Engineering, Hefei University of Technology, Hefei 230009, China. ${ }^{2}$ Department of Automation, University of Science and Technology of China, Hefei 230027, China. ${ }^{3}$ Department of Electrome- chanical Engineering, Faculty of Science and Technology, University of Macau, Macau, China.}

\section{Authors' Information}

Sen Qian, born in 1988, is currently a lecturer at School of Mechanical Engineering, Hefei University of Technology, China. He received his PhD degree from China University of Mining and Technology, China, in 2015. His research interests include robotics and automation.

Bin Zi, born in 1975, is currently a professor, the Dean of School of Mechanical Engineering, and the Director of Robotics Institute, Hefei University of Technology, China. He received his PhD degree from Xidian University, China, in 2007. His research interests include robotics and automation, mechatronics, and multirobot systems.

Wei-Wei Shang, born in 1981, is currently an associate professor at Department of Automation, University of Science and Technology of China. He received his PhD degree from University of Science and Technology of China, in 2008. His research interests include parallel robots, humanoid robots and robot vision.

Qing-Song Xu, born in 1978, is the Director of Smart and Micro/Nano Systems Laboratory and an associate professor of electromechanical engineering at the University of Macau, China. His current research area involves control and automation, MEMS-based micro/nano mechatronics and systems, and applications of computational intelligence. He is a Senior Member of IEEE and a Technical Editor of IEEE/ASME Transactions on Mechatronics.

\section{Competing Interests}

The authors declare that they have no competing interests.

\section{Funding}

Supported by National Natural Science Foundation of China (Grant Nos. 51605126, 51575150, 91748109)

\section{Publisher's Note}

Springer Nature remains neutral with regard to jurisdictional claims in published maps and institutional affiliations.

Received: 23 March 2018 Accepted: 6 August 2018

Published online: 17 August 2018

\section{References}

[1] Y Wiscchnitzer, N Shvalb, M Shoham. Wire-driven parallel robot: permitting collisions between wires. The International Journal of Robotics Research, 2008, 27(9): 1007-1026.

[2] B Zi, B Y Duan, J L Du, et al. Dynamic modeling and active control of a cable-suspended parallel robot. Mechatronics, 2008, 18(1): 1-12.

[3] THeyden, CWoernle. Dynamics and flatness-based control of a kinematically undetermined cable suspension manipulator. Multibody System Dynamics, 2006, 16(2): 155-177.

[4] A Berti, J P Merlet, M Carricato. Solving the direct geometrico-static problem of underconstrained cable-driven parallel robots by interval analysis. The International Journal of Robotics Research, 2016, 35(6): 723-739.

[5] B Zi, S Qian. Design, analysis and control of cable-suspended parallel robots and its applications. Singapore: Springer Singapore, 2017.

[6] X Q Tang. An overview of the development for cable-driven parallel manipulator. Advances in Mechanical Engineering, 2014, 2014(1): 1-9.

[7] P Dion-Gauvin, C Gosselin. Trajectory planning for the static to dynamic transition of point-mass cable-suspended parallel mechanisms. Mechanism and Machine Theory, 2017, 113: 158-178.

[8] S R Oh, S K Agrawal. Cable suspended planar robots with redundant cables: controllers with positive tensions. IEEE Transactions on Robotics, 2005, 3: 457-465

[9] A James, B Roger, D Nicholas. The NIST robocrane. Journal of Robotic Systems, 1993, 10: 709-724.

[10] R Bostelman, J Albus, N Dagalakis, et al. Applications of the NIST robocrane. Proceedings of International Symposium on Robotics and Manufacturing Maui Hi. 1994: 14-18.

[11] M Tanaka, Y Seguchi, S Shimada. Kineto-statics of skycam-type wire transport system. Proceedings of USA-Japan Symposium on Flexible Automation, Crossing Bridges: Advances in Flexible Automation and Robotics, 1988: 689-694.

[12] B Y Duan. A new design project of the line feed structure for large spherical radio telescope and its nonlinear dynamic analysis. Mechatronics, 1999, 9(1): 53-64.

[13] R D Nan. Five hundred meter aperture spherical radio telescope (FAST). Science in China, 2006, 49(2): 129-148.

[14] B Zi, J Lin, S Qian. Localization, obstacle avoidance planning and control of a cooperative CDPR for multiple mobile cranes. Robotics and ComputerIntegrated Manufacturing, 2015, 34: 105-123.

[15] S Seriani, P Gallina, A Wedler. A modular cable robot for inspection and light manipulation on celestial bodies. Acta Astronautica, 2016, 123: 145-153.

[16] M J Varela, M Ceccarelli, P Flores. A kinematic characterization of human walking by using CaTraSys. Mechanism and Machine Theory, 2015, 86: 125-139.

[17] Y Mao, X Jin, G G Dutta, et al. Human movement training with a cable driven arm exoskeleton (CAREX). IEEE Transactions on Neural Systems and Rehabilitation Engineering, 2015, 23(1): 84-92. 
[18] A M Pinto, E Moreira, J Lima, et al. A cable-driven robot for architectural constructions: a visual-guided approach for motion control and pathplanning. Autonomous Robots, 2017, 41(7): 1487-1499.

[19] E Barnett, C Gosselin. Large-scale 3D printing with a cable-suspended robot. Additive Manufacturing, 2015, 7: 27-44

[20] M J Varela, M Ceccarelli, P Flores. A kinematic characterization of human walking by using CaTraSys. Mechanism and Machine Theory, 2015, 86(86): 125-139.

[21] D Zanotto, G Rosati, S Minto, et al. Sophia-3: a semiadaptive cable-driven rehabilitation device with a tilting working plane. IEEE Transactions on Robotics, 2014, 30(4): 974-979.

[22] R Verhoeven. Analysis of the workspace of tendon-based Stewart platforms. Duisburg: Department of Mechanical Engineering, University of Duisburg-Essen, 2004.

[23] H W Liu, C Gosselin, T Laliberte. Conceptual design and static analysis of novel planar spring-loaded cable-loop-driven parallel mechanisms. Journal of Mechanisms and Robotics-Transactions of the ASME, 2012, 4(2): 11.

[24] K Azizian, P Cardou. The dimensional synthesis of planar parallel cabledriven mechanisms through convex relaxations. Journal of Mechanisms and Robotics-Transactions of the ASME, 2012, 4(3): 13.

[25] K Azizian, P Cardou. The dimensional synthesis of spatial cable-driven parallel mechanisms. Journal of Mechanisms and Robotics-Transactions of the ASME, 2013, 5(4): 8.

[26] L Gagliardini, S Caro, M Gouttefarde, et al. Discrete reconfiguration planning for Cable-Driven Parallel Robots. Mechanism and Machine Theory, 2016, 100: 313-337.

[27] B Zi, H H Sun, D Zhang. Design, analysis and control of a winding hybriddriven CDPR. Robotics and Computer-Integrated Manufacturing, 2017, 48: 196-208.

[28] Q M Jiang, V Kumar. Determination and stability analysis of equilibrium configurations of objects suspended from multiple aerial robots. Journal of Mechanisms and Robotics-Transactions of the ASME, 2012, 4(2): 21.

[29] S P Donohoe, S A Velinsky, T A Lasky. Mechatronic implementation of a force optimal underconstrained planar cable robot. IEEE-ASME Transactions on Mechatronics, 2016, 21(1): 69-78.

[30] A Capua, A Shapiro, S Shoval. SpiderBot: a cable-suspended walking robot. Mechanism and Machine Theory, 2014, 82: 56-70.

[31] S Qian, B Zi, H F Ding. Dynamics and trajectory tracking control of cooperative multiple mobile cranes. Nonlinear Dynamics, 2016, 83(1-2): 89-108.

[32] B Zi, S Qian, A Kecskeméthy. Design and development of a reconfigurable CDPR. The 14th IFTOMM World Congress, Taipei, Taiwan, China, October 25-30. 2015: 438-443

[33] X Dong, M Raffles, S C Guzman, et al. Design and analysis of a family of snake arm robots connected by compliant joints. Mechanism and Machine Theory, 2014, 77: 73-91.

[34] J W Suh, KY Kim, J W Jeong, et al. Design considerations for a hyperredundant pulleyless rolling joint with elastic fixtures. IEEE-ASME Transactions on Mechatronics, 2015, 20(6): 2841-2852.

[35] X Cui, W H Chen, X Jin, et al. Design of a 7-dof cable-driven arm exoskeleton (CAREX-7) and a controller for dexterous motion training or assistance. IEEE-ASME Transactions on Mechatronics, 2017, 22(1): 161-172.

[36] Y Mao, S K Agrawal. Design of a Cable-driven arm exoskeleton (CAREX) for neural rehabilitation. IEEE Transactions on Robotics, 2012, 28(4): 922-931.

[37] XW Zhao, B Zi, L Qian. Design, analysis, and control of a cable-driven parallel platform with a pneumatic muscle active support. Robotica, 2017, 35(4): 744-765.

[38] BT Gao, J Xu, J G Zhao, et al. A humanoid neck system featuring low motion-noise. Journal of Intelligent and Robotic Systems, 2012, 67(2): 101-116.

[39] D Lau, D Oetomo, S K Halgamuge. Generalized modeling of multilink cable-driven manipulators with arbitrary routing using the cable-routing matrix. IEEE Transactions on Robotics, 2013, 29(5): 1102-1113.

[40] B T Gao, ZY Zhu, J G Zhao, et al. Inverse kinematics and workspace analysis of a 3 dof flexible parallel humanoid neck robot. Journal of Intelligent and Robotic Systems, 2017, 87(2): 211-229.

[41] A Berti, J P Merlet, M Carricato. Solving the direct geometrico-static problem of underconstrained cable-driven parallel robots by interval analysis. International Journal of Robotics Research, 2016, 35(6): 723-739.
[42] M Carricato, J P Merlet. Stability analysis of underconstrained cable-driven parallel robots. IEEE Transactions on Robotics, 2013, 29(1): 288-296.

[43] M Carricato. Direct geometrico-static problem of underconstrained cable-driven parallel robots with three cables. Journal of Mechanisms and Robotics-Transactions of the ASME, 2013, 5(3): 10.

[44] Q M Jiang, V Kumar. The inverse kinematics of cooperative transport with multiple aerial robots. IEEE Transactions on Robotics, 2013, 29(1): 136-145.

[45] S R Oh, J C Ryu, P D Students, et al. Dynamics and control of a helicopter carrying a payload using a cable-suspended robot. Journal of Mechanical Design, 2015, 128(5): 1113-1121.

[46] B Zi, B Zhou. A modified hybrid uncertain analysis method for dynamic response field of the LSOAAC with random and interval parameters. Journal of Sound and Vibration, 2016, 374: 111-137.

[47] E Kljuno, LW li R. Vehicle simulation system: controls and virtual-realitybased dynamics simulation. Journal of Intelligent and Robotic Systems, 2008, 52(1): 79-99.

[48] B Zi, Z C Zhu, J L Du. Analysis and control of the cable-supporting system including actuator dynamics. Control Engineering Practice, 2011, 19(5): 491-501.

[49] J L Du, H Bao, C Z Cui, et al. Dynamic analysis of CDPRs with timevarying cable lengths. Finite Elements in Analysis and Design, 2012, 48(1): 1392-1399.

[50] J L Du, C Z Cui, H Bao, et al. Dynamic analysis of CDPRs using a variable length finite element. Journal of Computational and Nonlinear Dynamics, 2015, 10(1): 011013

[51] M A Khosravi, H D Taghirad. Dynamic modeling and control of parallel robots with elastic cables: singular perturbation approach. IEEE Transactions on Robotics, 2014, 30(3): 694-704.

[52] D Lau, D Oetomo, S K Halgamuge. Inverse dynamics of multilink cabledriven manipulators with the consideration of joint interaction forces and moments. IEEE Transactions on Robotics, 2015, 31(2): 479-488.

[53] H S Wang, C Wang, W D Chen, et al. Three-dimensional dynamics for cable-driven soft manipulator. IEEE-ASME Transactions on Mechatronics, 2017, 22(1): 18-28.

[54] J Eden, D Lau, Y Tan, et al. Available acceleration set for the study of motion capabilities for cable-driven robots. Mechanism and Machine Theory, 2016, 105: 320-336.

[55] S Abdolshah, D Zanotto, G Rosati, et al. Optimizing stiffness and dexterity of planar adaptive cable-driven parallel robots. Journal of Mechanisms and Robotics, 2017, 9(3): 031004

[56] M Gouttefarde, J F Collard, N Riehl, et al. Geometry selection of a redundantly actuated cable-suspended parallel robot. IEEE Transactions on Robotics, 2017, 31(2): 501-510.

[57] B L Wen, G Yang, HY Song, et al. A generic force-closure analysis algorithm for CDPRs. Mechanism and Machine Theory, 2011, 46(9): 1265-1275.

[58] B P Cong, HY Song, G Yang, et al. Force-closure workspace analysis of cable-driven parallel mechanisms. Mechanism and Machine Theory, 2006, 41(1): 53-69.

[59] M Gouttefarde, D Daney, J P Merlet. Interval-analysis-based determination of the wrench-feasible workspace of parallel cable-driven robots. IEEE Transactions on Robotics, 2011, 27(1): 1-13.

[60] D Lau, D Oetomo, S K Halgamuge. Wrench-closure workspace generation for CDPRs using a hybrid analytical-numerical approach. Journal of Mechanical Design, 2011, 133(7): 71004.

[61] K Azizian, P Cardou, B Moore. Classifying the boundaries of the wrenchclosure workspace of planar parallel cable-driven mechanisms by visual inspection. Journal of Mechanisms and Robotics-Transactions of the ASME, 2012,4(2): 5

[62] Q J Duan, V Vashista, S K Agrawal. Effect on wrench-feasible workspace of cable-driven parallel robots by adding springs. Mechanism and Machine Theory, 2015, 86: 201-210.

[63] B Ouyang, WW Shang. A new computation method for the force-closure workspace of CDPRs. Robotica, 2015, 33(3): 537-547.

[64] S H Yeo, G Yang, W B Lim. Design and analysis of cable-driven manipulators with variable stiffness. Mechanism and Machine Theory, 2013, 69: 230-244.

[65] HYuan, E Courteille, D Deblaise. Static and dynamic stiffness analyses of cable-driven parallel robots with non-negligible cable mass and elasticity. Mechanism and Machine Theory, 2015, 85: 64-81. 
[66] M Arsenault. Workspace and stiffness analysis of a three-DOF spatial cable-suspended parallel mechanism while considering cable mass. Mechanism and Machine Theory, 2013, 66: 1-13.

[67] J B Izard, A Dubor, P E Hervé, et al. Large-scale 3D printing with cabledriven parallel robots. Construction Robotics, 2017, 1(1-4): 69-76.

[68] Q Z Chen, W H Chen, G L Yang, et al. An integrated two-level self-calibration method for a cable-driven humanoid arm. IEEE Transactions on Automation Science and Engineering, 2013, 10(2): 380-391.

[69] H Jamshidifar, A Khajepour, B Fidan, et al. Kinematically-constrained redundant cable-driven parallel robots: modeling, redundancy analysis, and stiffness optimization. IEEE-ASME Transactions on Mechatronics, 2017, 22(2): 921-930.

[70] J L Du, H Bao, C Z Cui. Stiffness and dexterous performances optimization of large workspace CDPRs. Advanced Robotics, 2014, 28(3): 187-196.

[71] J T Bryson, X Jin, S K Agrawal. Optimal design of cable-driven manipulators using particle swarm optimization. Journal of Mechanisms and Robotics-Transactions of the ASME, 2016, 8(4): 8.

[72] B Ouyang, W W Shang. Wrench-feasible workspace based optimization of the fixed and moving platforms for CDPRs. Robotics and ComputerIntegrated Manufacturing, 2014, 30(6): 629-635.

[73] M Yamamoto, N Yanai, A Mohri. Trajectory control of incompletely restrained parallel-wire-suspended mechanism based on inverse dynamics. IEEE Transactions on Robotics, 2004, 20(5): 840-850.

[74] G Meunier, B Boulet, M Nahon. Control of an overactuated cable-driven parallel mechanism for a radio telescope application. IEEE Transactions on Control Systems Technology, 2009, 17(5): 1043-1054.

[75] H Kino, T Yahiro, F Takemura, et al. Robust PD control using adaptive compensation for completely restrained parallel-wire driven robots: translational systems using the minimum number of wires under zerogravity condition. IEEE Transactions on Robotics, 2007, 23(4): 803-812.

[76] M Gouttefarde, L Johann, R Christopher, et al. A Versatile Tension Distribution Algorithm for $n$-DOF Parallel Robots Driven by $n+2$ Cables. IEEE Transactions on Robotics, 2015, 31(6):1444-1457.

[77] J Yang, H Su, Z J Li, et al. Adaptive control with a fuzzy tuner for cablebased rehabilitation robot. International Journal of Control Automation and Systems, 2016, 14(3): 865-875.

[78] T C Wang, S C Tong, J Q Yi, et al. Adaptive inverse control of cable-driven parallel system based on type-2 fuzzy logic systems. IEEE Transactions on Fuzzy Systems, 2015, 23(5): 1803-1816.
[79] R Babaghasabha, M A Khosravi, H D Taghirad. Adaptive robust control of fully-constrained cable driven parallel robots. Mechatronics, 2015, 25: 27-36.

[80] S Abdelaziz, L Barbe, P Renaud, et al. Control of cable-driven manipulators in the presence of friction. Mechanism and Machine Theory, 2017, 107: 139-147.

[81] X Q Tang, Z F Shao. Trajectory generation and tracking control of a multi-level hybrid support manipulator in FAST. Mechatronics, 2013, 23(8): 1113-1122.

[82] J Fink, N Michael, S Kim, et al. Planning and control for cooperative manipulation and transportation with aerial robots. The International Journal of Robotics Research, 2011, 30(3): 324-334.

[83] L Barbazza, F Oscari, S Minto, et al. Trajectory planning of a suspended cable driven parallel robot with reconfigurable end effector. Robotics and Computer-Integrated Manufacturing, 2017, 48: 1-11.

[84] E Barnett, C Gosselin. Time-optimal trajectory planning of cable-driven parallel mechanisms for fully specified paths with $\mathrm{G}(1)$-discontinuities. Journal of Dynamic Systems Measurement and Control-Transactions of the ASME, 2015, 137(7): 12.

[85] C Gosselin, S Foucault. Dynamic point-to-point trajectory planning of a two-DOF cable-suspended parallel robot. IEEE Transactions on Robotics, 2014, 30(3): 728-736.

[86] X L Jiang, C Gosselin. Dynamic point-to-point trajectory planning of a three-DOF cable-suspended parallel robot. IEEE Transactions on Robotics, 2016, 32(6): 1550-1557.

[87] N Zhang, W W Shang, S Cong. Geometry-based trajectory planning of a 3-3 cable-suspended parallel robot. IEEE Transactions on Robotics, 2017, 33(2): 484-491.

[88] N Zhang, W W Shang. Dynamic trajectory planning of a 3-DOF underconstrained cable-driven parallel robot. Mechanism and Machine Theory, 2016, 98: 21-35

[89] L W Tang, X Q Tang, X L Jiang, et al. Dynamic trajectory planning study of planar two-DOF redundantly actuated cable-suspended parallel robots. Mechatronics, 2015, 30: 187-197.

[90] M H Korayem, H Tourajizadeh, A Zehfroosh, et al. Optimal path planning of a cable-suspended robot with moving boundary using optimal feedback linearization approach. Nonlinear Dynamics, 2014, 78(2): 1515-1543.

\section{Submit your manuscript to a SpringerOpen ${ }^{\circ}$ journal and benefit from:}

- Convenient online submission

- Rigorous peer review

- Open access: articles freely available online

- High visibility within the field

- Retaining the copyright to your article

Submit your next manuscript at $\boldsymbol{\nabla}$ springeropen.com 